\title{
Mechanism Assay of Honeysuckle for Heat-Clearing Based on Metabolites and Metabolomics
}

\author{
Hechen Wang ${ }^{1}$, Lu Tian ${ }^{1}$, Yiman Han ${ }^{1}$, Xiaoyao Ma ${ }^{1,2}$, Yuanyau Hou ${ }^{1,2, *}$ and Gang Bai ${ }^{1,2, *}$ \\ 1 State Key Laboratory of Medicinal Chemical Biology, College of Pharmacy, Nankai University, \\ Tianjin 300353, China; wang210723@163.com (H.W.); chrissy0708@163.com (L.T.); \\ hanyiman163@163.com (Y.H.); maxiaoyao@nankai.edu.cn (X.M.) \\ 2 Tianjin Key Laboratory of Molecular Drug Research, Nankai University, Tianjin 300353, China \\ * Correspondence: Houyy@nankai.edu.cn (Y.H.); gangbai@nankai.edu.cn (G.B.)
}

Citation: Wang, H.; Tian, L.; Han, Y.; Ma, X.; Hou, Y.; Bai, G. Mechanism Assay of Honeysuckle for Heat-Clearing Based on Metabolites and Metabolomics. Metabolites 2022, 12, 121. https://doi.org/10.3390/ metabo12020121

Academic Editor: Hunter N. B. Moseley

Received: 9 December 2021 Accepted: 26 January 2022 Published: 27 January 2022

Publisher's Note: MDPI stays neutral with regard to jurisdictional claims in published maps and institutional affiliations.

Copyright: (C) 2022 by the authors. Licensee MDPI, Basel, Switzerland. This article is an open access article distributed under the terms and conditions of the Creative Commons Attribution (CC BY) license (https:// creativecommons.org/licenses/by/ $4.0 /)$.

\begin{abstract}
Nonsteroidal anti-inflammatory drugs (NSAIDs), such as cyclooxygenase (Cox)-1/2 inhibitor, have emerged as potent antipyretics and analgesics. However, few herbs with Cox- $1 / 2$ inhibitory activity are commonly used for heat-clearing in China. Although these are known to have antipyretic activity, there is a lack of molecular data supporting their activity. Using the traditional Chinese medicine herb honeysuckle (Hon) as an example, we explored key antipyretic active compounds and their mechanisms of action by assessing their metabolites and metabolomics. Mitogen-activated protein kinase (MAPK) 3 and protein kinase B (AKT) 1 were suggested as key targets regulated primarily by chlorogenic acid (CA) and swertiamarin (SWE). CA and SWE synergistically inhibited the production of interleukin (IL)-1 and IL-6, alleviated generation of prostaglandin E2, and played an antipyretic role equivalent to honeysuckle extract at the same dose contents within $3 \mathrm{~h}$. Collectively, these findings indicated that lipopolysaccharide-induced fever can be countered by CA with SWE synergistically, allowing the substitution of a crude extract of complex composition with active compounds. Our findings demonstrated that, unlike the traditional NSAIDs, the Hon extract showed a remote and indirect mechanism for alleviating fever that depended on the phosphatidylinositol-3-kinase-AKT and MAPK pathways by regulating the principal mediator of inflammation.
\end{abstract}

Keywords: metabolites; metabolomics; chlorogenic acid; swertiamarin; antipyretic; anti-inflammatory

\section{Introduction}

Starting with aspirin, nonsteroidal anti-inflammatory drugs (NSAIDs), which target cyclooxygenase (COX)-1/2, have emerged as potent antipyretics and analgesics [1] However, almost no heat-clearing herbal medicines containing COX-1/2 inhibitors based on traditional Chinese medicine are being used for alleviating fever. One of the few examples of plants that are being used is honeysuckle (Hon), which refers to the flower buds of Lonicera japonica Thunb., possessing as it does potential anti-pyretic and antiinflammatory activities, and which is widely used clinically after extraction [2]. So far, more than 140 phytoconstituents have been identified from honeysuckle extracts. In addition to essential oils, an abundance of flavones, organic acids, iridoids, and triterpenoid saponins have also been found [3]. However, the key component responsible for its antipyretic activity and its corresponding target remain unknown. Therefore, it is important to investigate the complex mechanism behind antipyretic activity exerted by these active compounds, as this will provide the knowledge required to precisely tailor therapies for various conditions, such as exopathogenic diseases, sores, carbuncles, and some infectious diseases.

Natural products (NPs) remain the most prolific source of inspiration for the development of drugs [4]. The novel modes of action exhibited by NPs have always made them a subject of great interest in medicine [5]. With the exploration of NPs has become more common, increased access to biological and chemical data, and the development of 
data analysis algorithms, and integration of computational methods in NP drug discovery pipelines may be expected to increase significantly [6]. Data pre-processing using convolutional neural networks reportedly performed better in peak alignment and identification from liquid chromatography-mass spectrometry (LC-MS), compound identification and quantification, and integration and interpretation of multi-omics data [7]. Considering the complex and elusive nature of botanical products and their metabolism in the human body, metabolomic data generated for herbal drugs are large compared to those for single compounds. Combining information from different sources, that is, chemical, biochemical, biological, and in silico, with advanced computer algorithms and effectively handling big data can open new possibilities for NP research [8]. Unfortunately, the integration of multi-omics data-those for the structures/components and for their targets-remains a challenge.

Here, we used honeysuckle as a model herb and its extract to screen the key bioactive ingredients and potential protein targets in order to explore the antipyretic mechanism based on metabolites and metabolomic data. LC-MS coupled with molecular networking $(\mathrm{MN})$ was adopted to analyze the prototype and metabolites in the blood and build a chemomic profiled network consisting of precursor ions. Virtual docking and annotation enrichment analyses were used to identify their structures and potential targets. Concurrently, a metabonomic assay was employed to detect the major reversal changes that were regulated by honeysuckle extractive preparation (HEP) administration in a lipopolysaccharide (LPS)-induced rat fever model. The interpretation of integrated data revealed chlorogenic acid (CA) and swertiamarin (SWE) as key compounds acting on both the phosphatidyl-inositol-3-kinase-protein kinase B (PI3K-AKT) and p38 mitogen-activated protein kinase (MAPK) pathways that are associated with fever.

\section{Results}

\subsection{Global Metabolic Profiling Coupled with MN Identifies the Key Metabolites}

Global metabolite profiling based on negative and positive ion modes was performed to identify key metabolites in plasma derived from HEP, a honeysuckle oral liquid freezedried powder. The MS data of different groups were integrated into the Global Natural Product Social Molecular Networking (GNPS) platform. As shown in Figure 1A, the MN map contained 1371 precursor ions, including 297 clusters (node $\geq 2$ ) and 313 single nodes. Interestingly, compared with the overlapped metabolite distribution in HEP- and HEPtreated plasma (Figure 1B), a total of eight main prototype components (P1-8) showed a significant difference in dysregulated metabolites. Based on the exact mass measurements and fragmentation patterns confirmed from references, as well as the Agilent natural product MN database, these compounds were identified as CA, SWE, cryptochlorogenic acid, rutin, secoxyloganin, 3,5-dicaffeoylquinic acid, 4,5-di-O-caffeoylquinic acid, and sweroside (detailed in Table 1), as shown in Figure 1C. The prototype-derived 37 metabolites (M1-37) linked by molecular weight profiling are listed in Table S1. The results showed that the potentially effective compounds were divided into three parts: polyphenol acids, flavonoids, and iridoids. Based on the $\mathrm{m} / \mathrm{z}$ intensity response (Figure 1B, bottom panel), the eight potentially effective compounds were selected and used for the following target prediction by the PharmMapper website.

Table 1. Related MS information of identified compound in HEP.

\begin{tabular}{|c|c|c|c|c|c|c|}
\hline Name & $t_{R}(\min )$ & $m / z$ & Formula & MS/MS $(m / z)$ & Fit Score & Identification \\
\hline P1 & 3.487 & 355.1027 & $\mathrm{C}_{16} \mathrm{H}_{18} \mathrm{O}_{9}$ & $\begin{array}{c}115.0277 \\
91.6649\end{array}$ & 99.7 & Chlorogenic acid \\
\hline $\mathrm{P} 2$ & 16.354 & 375.1284 & $\mathrm{C}_{16} \mathrm{H}_{22} \mathrm{O}_{10}$ & $\begin{array}{l}121.6451 \\
137.7673\end{array}$ & 99.8 & Swertiamarin \\
\hline P3 & 4.372 & 355.1027 & $\mathrm{C}_{16} \mathrm{H}_{18} \mathrm{O}_{9}$ & $\begin{array}{c}84.399 \\
154.1601\end{array}$ & 99.7 & Cryptochlorogenic acid \\
\hline
\end{tabular}


Table 1. Cont.

\begin{tabular}{|c|c|c|c|c|c|c|}
\hline Name & $t_{R}(\min )$ & $m / z$ & Formula & MS/MS $(m / z)$ & Fit Score & Identification \\
\hline $\mathrm{P} 4$ & 14.265 & 611.1608 & $\mathrm{C}_{27} \mathrm{H}_{30} \mathrm{O}_{16}$ & $\begin{array}{c}63.9512 \\
360.5813\end{array}$ & 99.8 & Rutin \\
\hline P5 & 10.242 & 405.1403 & $\mathrm{C}_{17} \mathrm{H}_{24} \mathrm{O}_{11}$ & $\begin{array}{l}68.8756 \\
124.453\end{array}$ & 99.8 & Secoxyloganin \\
\hline P6 & 7.249 & 516.4517 & $\mathrm{C}_{25} \mathrm{H}_{24} \mathrm{O}_{12}$ & $\begin{array}{c}77.3744 \\
248.0019\end{array}$ & 99.8 & 3,5-dicaffeoyl qunic acid \\
\hline P7 & 8.225 & 355.1027 & $\mathrm{C}_{16} \mathrm{H}_{18} \mathrm{O}_{9}$ & $\begin{array}{c}82.6558 \\
329.2093\end{array}$ & 99.7 & $\begin{array}{l}\text { 4,5-Di-O-caffeoyl quinic } \\
\text { acid }\end{array}$ \\
\hline P8 & 12.347 & 359.1334 & $\mathrm{C}_{16} \mathrm{H}_{22} \mathrm{O}_{9}$ & $\begin{array}{c}62.7029 \\
242.1572\end{array}$ & 99.8 & Sweroside \\
\hline
\end{tabular}
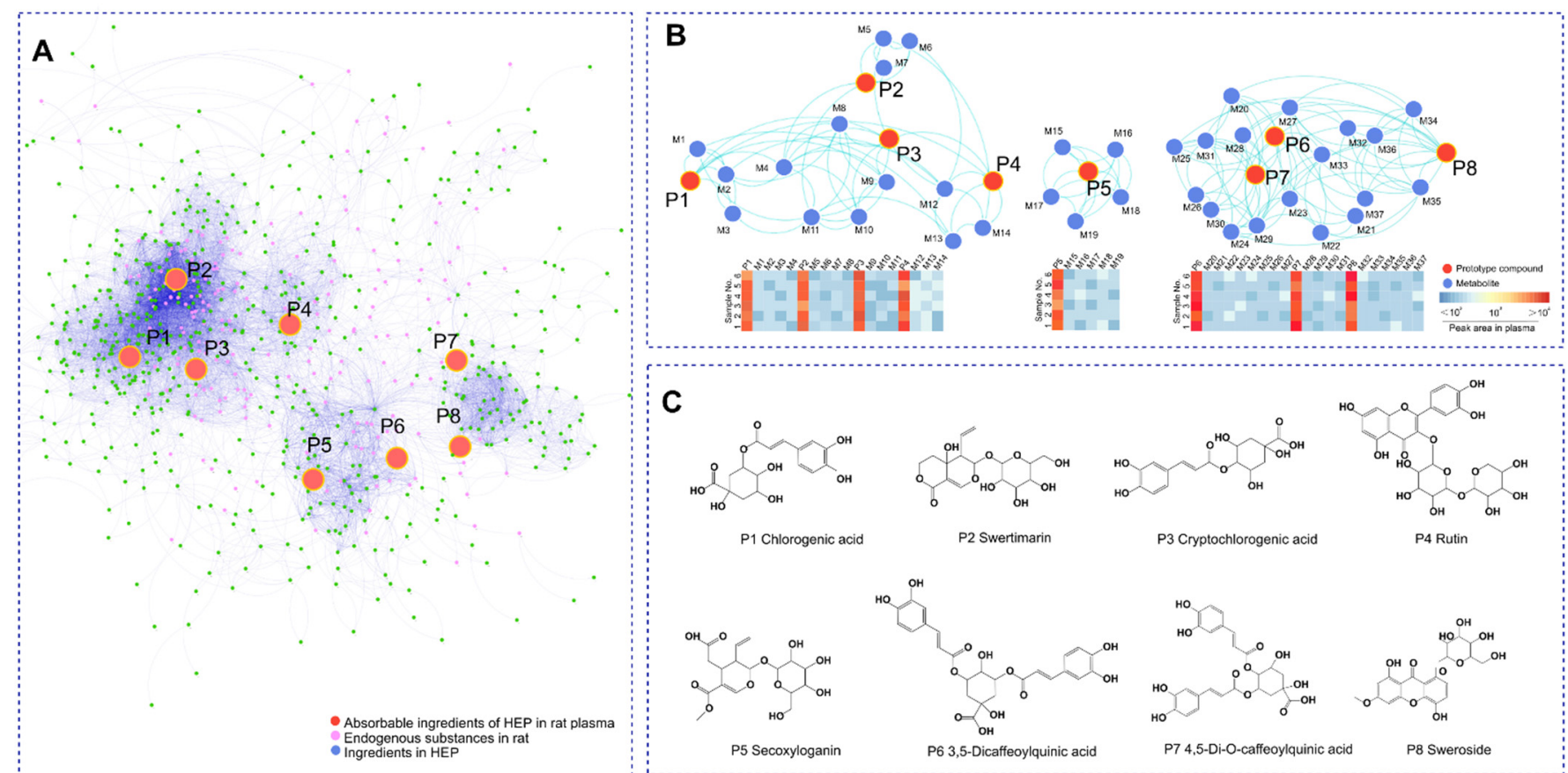

Figure 1. The identification of absorbable ingredients in HEP based on the MN assay. (A) Whole MN profiling of HEP, blank plasma sample, and administration plasma sample after oral HEP. (B) The network association diagram showed a clustering of the absorbable ingredients and their metabolites (top panel), as well as the relative contents of the absorbable ingredients and their metabolites. The minimum concentration was expressed as dark blue and the maximum concentration was expressed as dark red (bottom panel). (C) The structure of the key prototype compounds.

\subsection{Integrated Analysis Reveals the Core Mechanism of Antipyretic Effects}

To explore the underlying molecular mechanisms, the corresponding plasma samples were subjected to metabolomic analysis by LC-MS. As shown in Figure 2A, 289 different metabolites out of the total 952 were found in the quantitation to have a fold-change of $\geq 1.29$ or $\leq 0.91$ and corrected $p$-value $\leq 0.05$. The detailed differential metabolites are shown in Figure 2B. According to the Venn diagram analysis, only 26 metabolites were identified in all the comparison groups (Figure 2C). A total of 23 pathways were annotated using KEGG pathway enrichment analysis (Figure 2D). Among these, eight key pathways namely, HIF-1, PI3K-AKT, MAPK, TNF, NF-kappa B, FoXo signaling pathways, inflammatory mediator regulation of TRP channels, and GABAergic synapse, were related to fever and inflammation. 

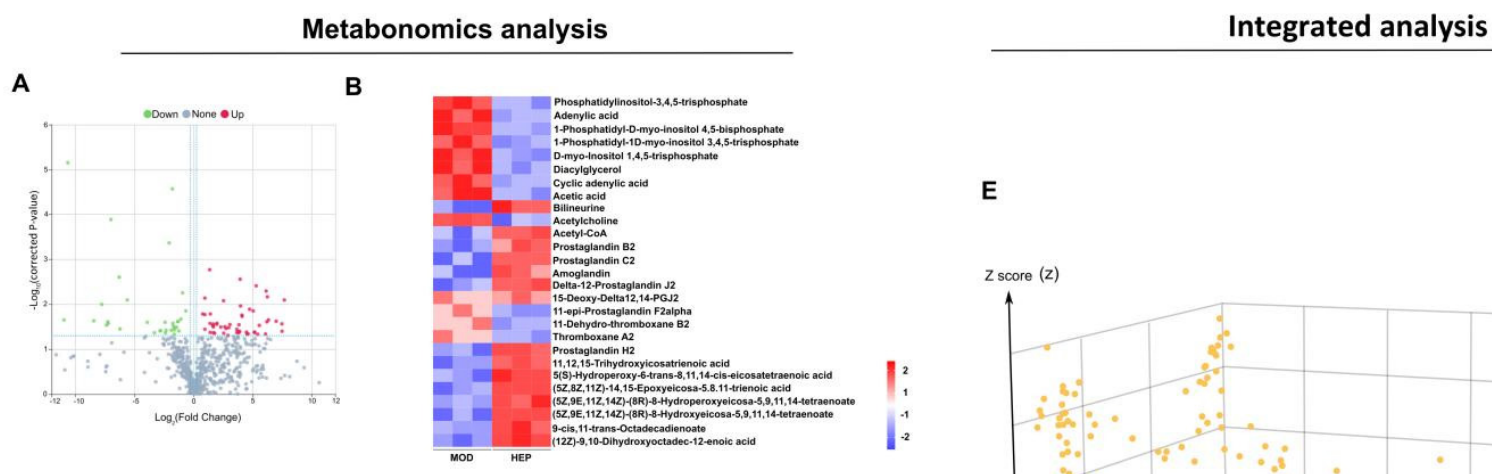

D
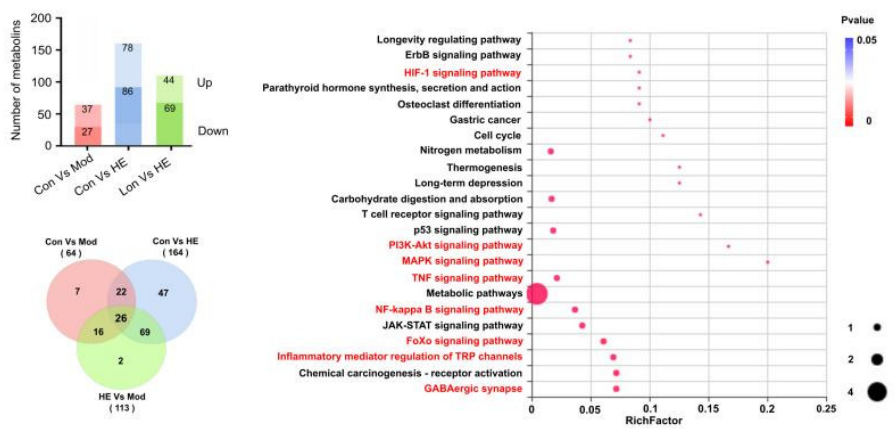

E

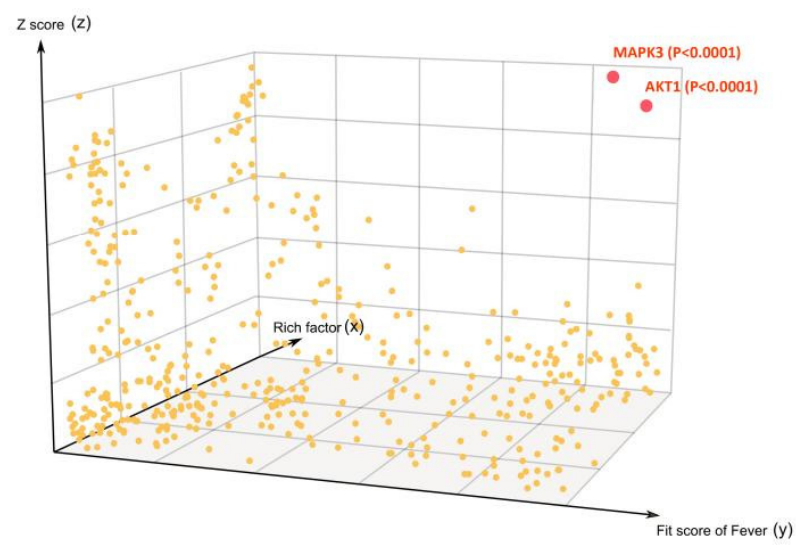

Figure 2. (A) Volcano plot of the quantitative metabolites in plasma after HEP administration according to fold change and corrected $p$-value. Metabolites with significant variation in abundance are shown as red (up) or blue (down) dots. (B) Heat map of cluster analysis illustrates the key related metabolites. (C) Metabonomic analysis of plasma. Statistical analysis of differential metabolites among experimental groups (top panel). Venn diagram analysis of the differential metabolites (bottom panel). (D) The 23 significantly enriched KEGG pathways. The $x$-axis shows rich factor; the $y$-axis corresponds to the KEGG pathway. The fever and inflammation pathways are shown in red. (E) The systematic integrative analysis of all the proteins associated with fever based on metabolites and metabolomics data. The x-axis represents the protein's fit factor for fever from GeneCards; the $y$-axis represents the protein's rich factor on the key pathway from KEGG; the z-axis represents the target protein's docking score rich from PharmMapper. Adjusted $p$-values of different correlation scores were used to enable robust statistical interpretation.

To understand the key action nodes of HEP in fever, all associated proteins were used in the integrated analysis involved in the fever-pathway proteins (273) enriched from the above metabonomics, target proteins (400) were predicted from the key chemicals via PharmMapper, and fever-associated proteins (653) were obtained from GeneCards. A 3D diagram was constructed based on the correlation scores. As shown in Figure 2E, based on metabolites and metabolomics assays and system integration, only two distinct differential targets were explored: MAPK3 (ERK1) and AKT1, regulated by CA and SWE, respectively (detailed in the Supplementary Materials).

\subsection{The Antipyretic Effects of CA Combined with SWE}

To verify whether the combination of CA and SWE played an alternative antipyretic role with HEP, the same dose of phenolic acids (CA $20 \mathrm{mg} / \mathrm{kg}$ ) and iridoid glycosides (SWE $10 \mathrm{mg} / \mathrm{kg}$ ) in HEP, which was replaced by Hon oral liquid, was used to evaluate the antipyretic effects in the LPS-induced rat fever model. As expected, LPS-induced fever can be significantly suppressed by aspirin. Similarly, the HEP and CA+SWE groups showed almost the same antipyretic effect within $3 \mathrm{~h}$ (Figure 3A, left panel). In the first half of the observation period, the effect of CA alone was better than that of SWE (Figure 3B, right panel). For a detailed comparison, the anal temperature of each group was compared at two peak times. Interestingly, there was no significant difference between the HEP and CA+SWE 
groups. Compared to the SWE group, CA had a better effect in the first $0.5 \mathrm{~h}(p<0.001)$.

The effect of SWE was better than that of CA at $2.25 \mathrm{~h}(p<0.05)$ (Figure 3C,D).

A

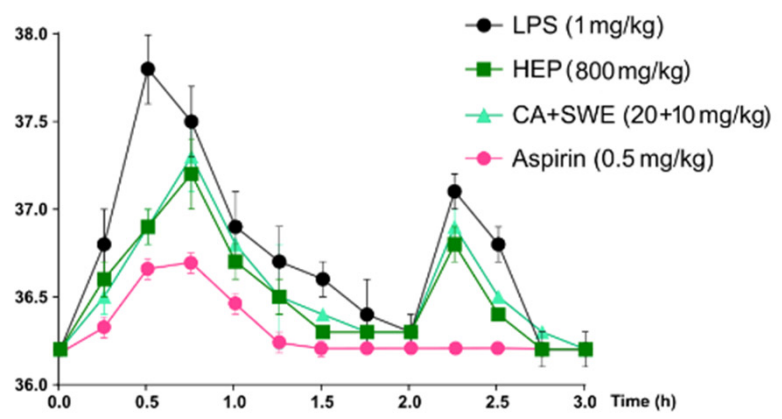

C

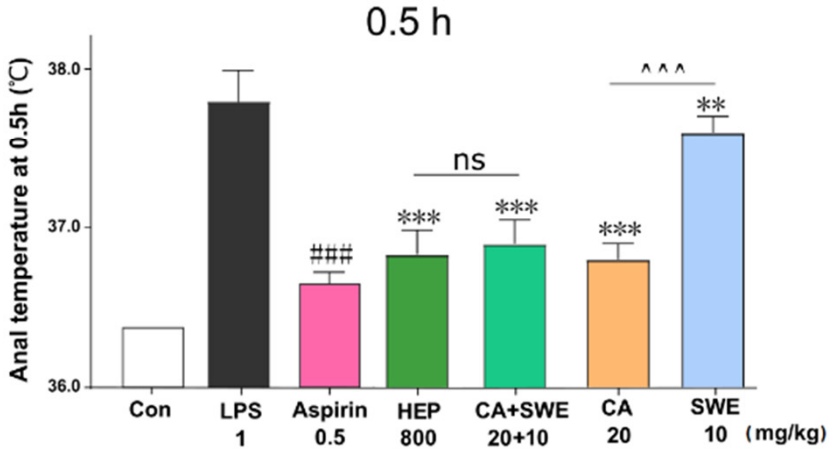

B

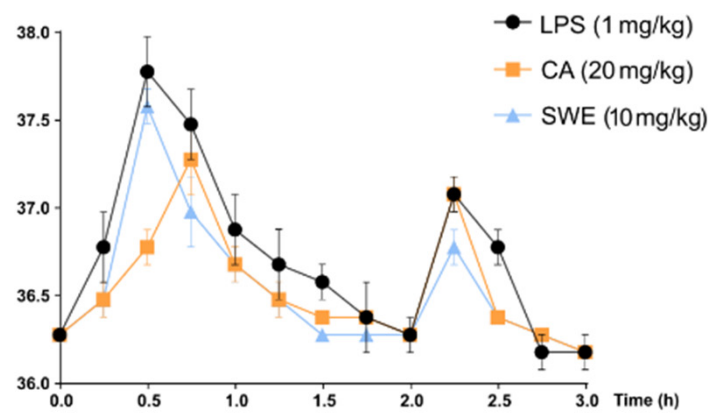

D

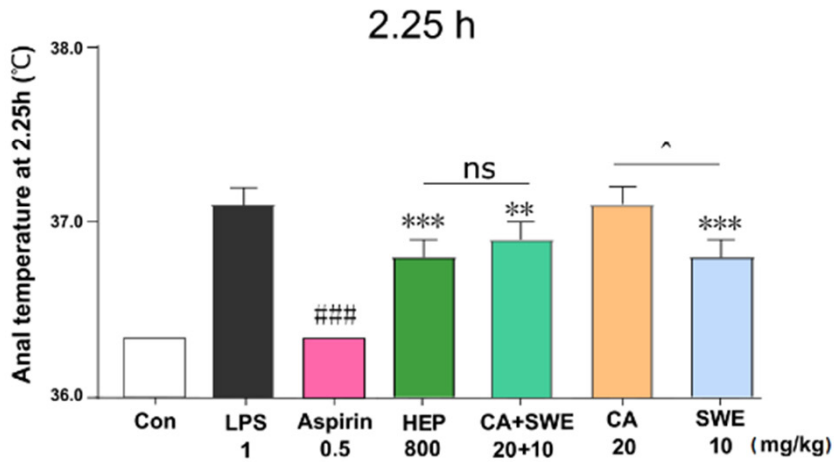

Figure 3. (A,B) Anal temperature curve of Aspirin, HEP, CA+SWE (left panel) and CA, SWE (right panel) in the first $3 \mathrm{~h}$ after intraperitoneal injection of LPS. Values are given as means \pm standard deviation (SD) $(n=10)$. (C,D) Anal temperature at $0.5 \mathrm{~h}$ and $2.25 \mathrm{~h}$ for each group. ${ }^{\# \#} p<0.001$, Aspirin vs. LPS; ${ }^{* *} p<0.01,{ }^{* * *} p<0.001$, drug intervention group vs. LPS; ${ }^{\wedge} p<0.05,{ }^{\wedge \wedge} p<0.001$, CA vs. SWE; ns, not significant $(n=10)$.

\subsection{The Potential Mechanism of CA and SWE against Fever}

To evaluate the effect of CA and SWE on the expression of downstream inflammatory factors by regulating MAPK3 and AKT1 targets, the expression of interleukin (IL)-1, IL-6, and prostaglandin E2 (PGE2) was also assessed. As shown in Figure 4A-C, compared to the LPS group, all groups showed significantly reduced levels of PGE2, IL-1, and IL-6. As expected, there was no significant difference between the HEP and CA+SWE groups $(p>0.05)$. Moreover, the result indicated that CA is better at downregulating IL-1 level than SWE, both at 0.5 and $2.25 \mathrm{~h}(p<0.01)$. It was suggested to target MAPK3 (ERK1) signaling. Extracellular signal-regulated protein kinases 1 and 2 (ERK1/2) are signal transducers of IL-1 $\beta$ via the p38 MAPK pathway in inflammation processes [9]. SWE exhibited an inhibitory effect on IL-6 at $0.5 \mathrm{~h}(p<0.001)$, which was consistent with previous findings indicating SWE inhibition of phosphorylation of AKT and alleviation of the PI3K-AKT signaling pathway [10]. Collectively, these findings suggest that the resultant LPS-induced fever can be rescued by the synergetic effect of CA with SWE and that the combined action of the p38-MAPK and PI3K-AKT pathways leads to a better antipyretic effect. 
A

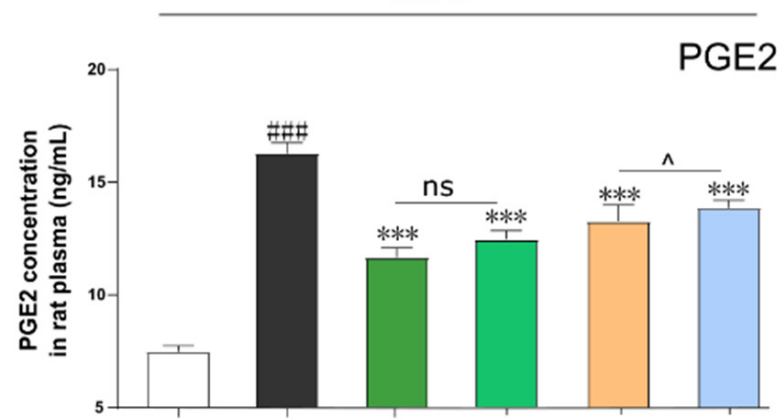

B

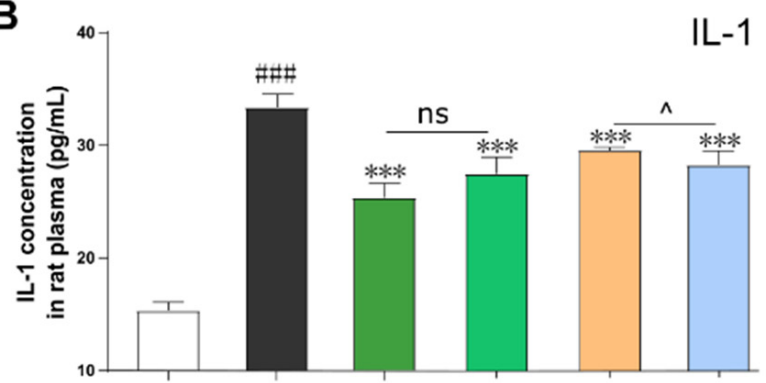

C

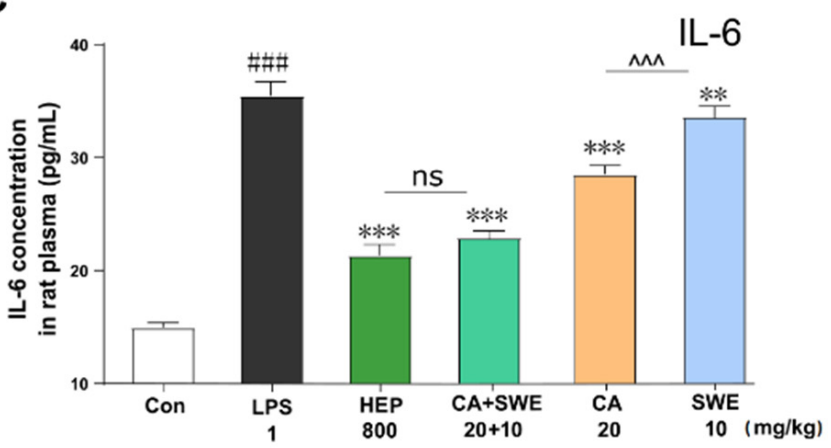

$2.25 \mathrm{~h}$
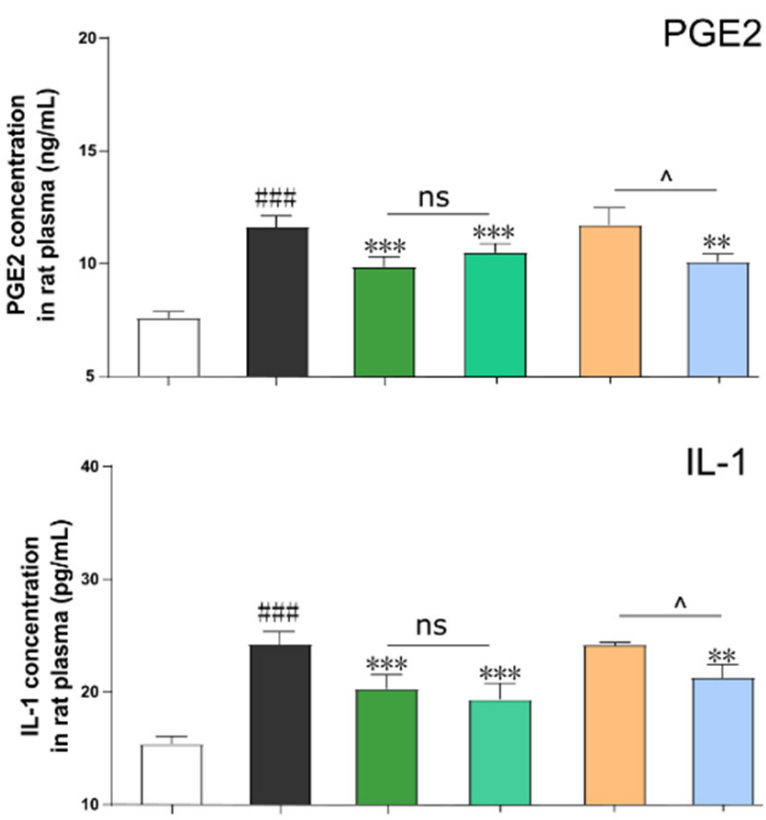

IL-6

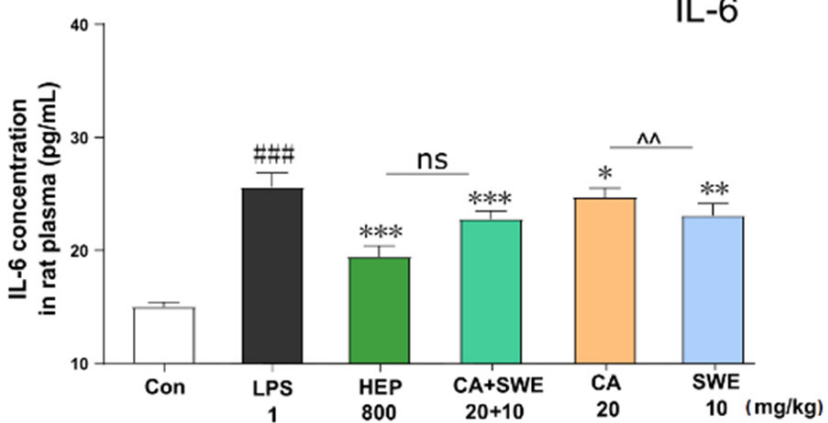

Figure 4. HEP, CA, and SWE downregulated the expression of pro-inflammatory cytokines in LPSinduced fever rats. The protein expression levels of PGE2 (A), IL-1 (B), and IL-6 (C) in the plasma at $0.5 \mathrm{~h}$ and $2.25 \mathrm{~h}$ were tested with ELISA kits. ${ }^{\# \#} p<0.001$, LPS vs. self-control; ${ }^{*} p<0.005,{ }^{* *} p<0.01$, ${ }^{* * *} p<0.001$, drug intervention group vs. LPS; ${ }^{\wedge} p<0.05,{ }^{\wedge} p<0.01,{ }^{\wedge} \wedge p<0.001$, CA vs. SWE; ns, not significant $(n=6)$

\section{Discussion}

Fever is an acute-phase complex physiological response by a host immune system against pathogens. The activation of peripheral immune cells by an infectious agent increases the generation of several inflammatory mediators. The first cytokine induced by immune challenge is TNF- $\alpha$, followed by IL-1, IL-6, and other cytokines [11]. Systemic inflammation is often modeled by administering bacterial LPS, which secretes several agents, triggering autonomic and behavioral thermo-effector responses that cause either fever or hypothermia [12]. In mammals, PGE2 is the principal mediator of fever. IL-1 and IL- 6 in the blood activate the expression of COX2 and PGS through their receptors on brain endothelial cells, evoking fever by eliciting PGE2 synthesis [13]. These PGE2 and pro-inflammatory cytokines induce an increase in body temperature.

As is widely known, the Toll-like receptor (TLR)-mediated PI3K-AKT and p38-MAPK pathways are involved in resisting pathogenic invasions. These pathways utilize transcription factors, such as NF-kB and AP-1, and further regulate the production of IL-1 and IL-6 [14]. Evidence suggests that the PI3K-Akt, MAPK, and NF-kB pathways participate in the inflammation process [15]. The extracellular signal-regulated kinases ERK1/2 
and p38 play key roles in the MAPK pathway. The phosphorylation of ERK $1 / 2$ and p38 promotes the production of TNF- $\alpha$, IL-6, and PGE2 [16]. Meanwhile, IL-1 $\beta$ induction of IL-6 production by activation of the $\mathrm{p} 38$ MAPK-NF- $\mathrm{kB}$ signaling pathway with a posttranscriptional mechanism [17], and the expression of IL-6, which is induced by IL- $1 \beta$, was significantly reduced by p38-MAPK inhibition [18]. Hence, inhibiting the expression of IL-1 can simultaneously alleviate IL-1-triggered IL-6 expression and protein secretion [19].

In principle, the absorbed components in vivo, having sufficiently prolonged exposure may be directly associated with therapeutic efficacy [20]. Therefore, identifying the key bioactive components via global metabolic profiling, while focusing on the pathological effects, may be meaningful. In contrast to classic NSAIDs, few COX-1/2 inhibitors have been identified in commonly used clinical antipyretic herbs. Owing to the large number of compounds in the herbs and limited information about the huge regulatory network, accurately determining the most bioactive compound, its target, as well as the mechanism of action, remains challenging [21].

Here, $\mathrm{MN}$ based on approximate hierarchical clustering was used to find the main exposure prototype or metabolites in the blood from HEP. Chrom Align Net algorithm, and Mass-MetaSite were used to match and identify these components. The uppermost bioactive compound was utilized for target prediction using PharmMapper. Integrated biological data interpretation benefited from multi-omics data conversion and the diseaseassociated target database can improve the possibilities for target discovery [22]. Hence, the multi-dimensional protein targets, including the antipyretic-related targets from GeneCards, metabolomics-enriched targets from HEP intervention, and molecular docking targets were integrated. Through our integrated analysis processes, CA and SWE were found to be the key ingredients. It was known that CA attenuated different stimuli-induced ulcerative colitis or toxicity through the MAPK-ERK-JNK or MAPK-AKT pathways $[23,24]$. SWE was demonstrated to target the AKT PH domain, deactivated the phosphorylation of AKT, and presented significant anti-inflammatory activity [10]. Organic acids were indicated as the dominant compounds responsible for anti-inflammatory effects [25]. The above evidence also supported our results from another aspect.

In conclusion, our findings indicate that $\mathrm{CA}$ targets MAPK3, regulating the expression of both IL-1 and IL-6 and playing a vital role compared to SWE, which acts on the PI3KAKT pathway to reduce the production of IL- 6 via the AKT target. By attenuating these principal pro-inflammatory cytokines and their driving PGE2 expression, CA with SWE synergistically can simulate the effect of HEP complexes for relieving fever. This paper provides a feasible solution by using metabolite and metabolomics analysis to uncover the associations of complex systems.

\section{Materials and Methods}

\subsection{Reagents and Chemicals}

The sample of HEP directly replaced by honeysuckle (Jinyinhua) oral liquid (lot no. 635031, each package $20 \mathrm{~mL}$, about $90 \mathrm{mg}$ crude drug per $\mathrm{ml}$ ) was produced by Zhenao Honeysuckle Pharmaceutical Co., Ltd. (Xianning, China). Chlorogenic acid (CA) and swertiamarin (SWE) (purity $>98 \%$ ) were procured from Meilunbio (Dalian, China). Aspirin was obtained from Solarbio (Beijing, China). Rat IL-6, IL-1 $\beta$, and PGE2 enzyme-linked immunosorbent assay kits were purchased from Thermo Fisher Scientific (Waltham, MA, USA) and LPS from Macklin (Shanghai, China). Moreover, LC-MS-grade methanol, acetonitrile, and formic acid $(99.5+\%)$ were purchased from Fisher Scientific, Inc. (Pittsburgh, PA, USA) and used for the preparation of mobile phases and solutions.

\subsection{Animals}

Experimental Sprague Dawley rats (8 weeks old, male, weighing $200 \pm 10 \mathrm{~g}$ ) were purchased from Beijing Vital River Laboratory Animal Technology Co., Ltd. (Beijing, China). All animals were fed a standard diet (Trophic Animal Feed High-tech Co. Ltd., 
Nantong, China) and given water ad libitum under controlled environmental conditions (temperature, $22 \pm 2{ }^{\circ} \mathrm{C}$; humidity, $45 \% \pm 5 \%$; light/dark cycle, $12 \mathrm{~h} / 12 \mathrm{~h}$ ).

After adaptation to the standard laboratory conditions for two weeks, the experimental rats were randomly allocated to the following groups $(n=10)$ : control group (Con), Mod group (LPS, $1 \mathrm{mg} / \mathrm{kg}$ ), Aspirin (0.5 mg/ $\mathrm{kg})$, HEP group ( $800 \mathrm{mg} / \mathrm{kg}$ crude drug), CA group $(20 \mathrm{mg} / \mathrm{kg})$, SWE group $(10 \mathrm{mg} / \mathrm{kg})$, and CA+SWE group $(20 \mathrm{mg} / \mathrm{kg}$ CA plus $10 \mathrm{mg} / \mathrm{kg}$ SWE). In addition to the Con group, all groups were established by administering intraperitoneal injections at the same dose of LPS $(1 \mathrm{mg} / \mathrm{kg})$. After LPS injection, intragastric administration of Aspirin, HEP, CA, SWE, or CA+SWE was carried out immediately. The rectal temperature was measured with a rectal probe coupled to a digital thermometer (Taimeng, Sichuan, China) every $15 \mathrm{~min}$ for $3 \mathrm{~h}$, and the temperature was calibrated for 15-30 s before readings were taken. All measurements were performed at a stable ambient temperature of $23 \pm 1{ }^{\circ} \mathrm{C}$.

\subsection{Plasma Sample Preparation}

First, plasma samples were obtained at different time points after oral administration of HEP $(800 \mathrm{mg} / \mathrm{kg})$. Blood samples were taken from retroorbital blood, and $100 \mu \mathrm{L}$ of frozen plasma samples were thawed and placed into centrifuge tubes $(1.5 \mathrm{~mL})$. The samples were then thoroughly mixed with $200 \mu \mathrm{L}$ of methanol and vortexed for $5 \mathrm{~min}$. The solutions were centrifuged at $8000 \mathrm{rpm} / \mathrm{min}$ for $10 \mathrm{~min}$. The supernatants at $0.5 \mathrm{~h}$ were filtered through a $0.22 \mu \mathrm{m}$ membrane for metabonomic analyses. Similarly, a plasma mixture at $0.15,0.5,1.0$, and $2.0 \mathrm{~h}$ was used for metabolite identification. Meanwhile, the plasma after administration was also collected at $0.5 \mathrm{~h}$ and $2.25 \mathrm{~h}$ and centrifuged at $8000 \mathrm{rpm} / \mathrm{min}$ for $10 \mathrm{~min}$. The supernatants were then used for PGE2, IL-1, and IL-6 analyses by enzyme-linked immunosorbent assay.

\subsection{UPLC-MS Analysis for Metabolites}

An Align 1290 Infinity II UPLC combined with the 6550 iFunnel quadrupole time of a flight LC-MS system was used in the proofing and identification of metabolites (the conditions of chromatographic and MS-MS are described in the Supplementary Materials). Chrom Align Net software was used to identify the metabolites [26].

\subsection{Data Processing}

A comprehensive approach, including global metabolic profiling by $\mathrm{MN}$ based on MS-MS coupled with a series of integrated analysis, was used to discover and identify key compounds. The workflow of functional ligand discovery from metabolic profiling and the elucidation of the antipyretic mechanism via metabolomic data was performed, as shown in Figure 5.

For molecular network construction, the MS-MS data for metabolites were collected and converted to mzXML format using Proteo Wizard software (www.proteowizard. sourceforge.net, Proteo Wizard, Palo Alto, CA, USA) and uploaded separately to the GNPS platform (https: / /gnps.ucsd.edu, UCSD, San Diego, CA, USA) (accessed on 16 November 2021). The GNPS parameters were set as follows: mass error of less than $0.02 \mathrm{Da}$, matched peaks greater than 6 , and cosine score greater than 0.50 . Next, the merged molecular network was successfully obtained according to our reported method [27]. Finally, Cytoscape software v 3.7.1 (www.cytoscape.org, NRNB, Hill St, San Diego, CA, USA) (accessed on 16 November 2021) was used to build the molecular network.

For metabolomic analyses, the operations were performed according to our previous study [21]. A Q-Exactive HF X mass spectrometer (Thermo Fisher Scientific, Waltham, MA, USA) was used for metabolite detection. After pretreatment of the rat plasma by deproteinization, Compound Discoverer software (version 3.0; Thermo Fisher Scientific, USA) was used for further data analysis, and the BGI Library, mzCloud, and ChemSpider databases were used as the search databases. 


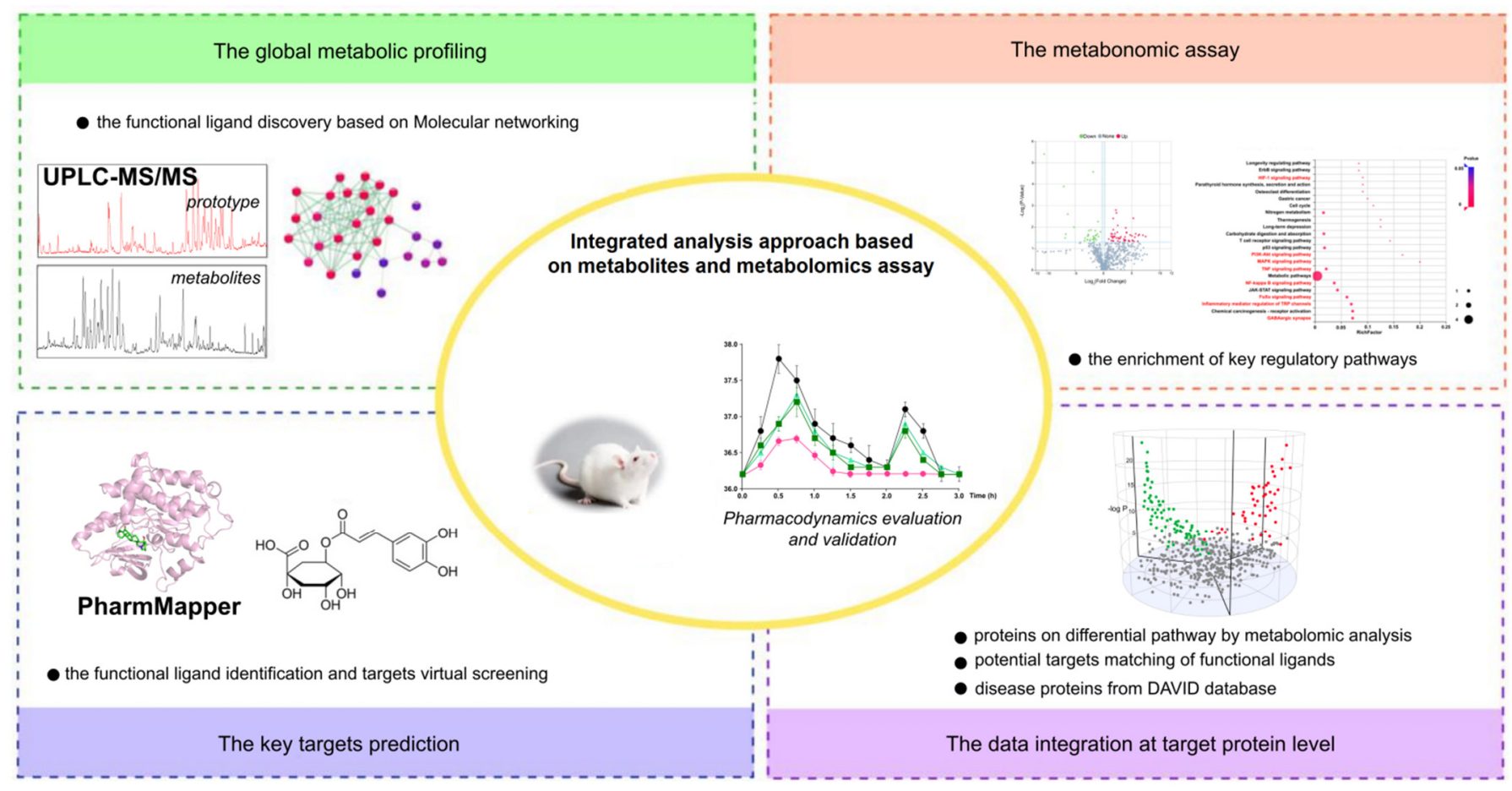

Figure 5. Workflow of the integrated analysis approach based on metabolites and metabolomics assay approach. The process including LC-MS combined MN screening to obtain the prototype compounds and its metabolites; the enrichment of the key target proteins from metabonomics assay; target prediction of potential bio-active compounds; and integrative analysis and validation of biological effect.

To identify the key targets, the PharmMapper database (http:/ / www.lilab-ecust.cn/ pharmmapper /) (accessed on 16 November 2021) was used to predict key metabolites from HEP [27]. Python was adopted for data integration of all proteins, including differential pathways by metabolomic analysis, potential targets matching by the PharmMapper, disease proteins associated with fever, and the DAVID database. The intersect protein was visually displayed using PEAC seq (https://peac.hpc.qmul.ac.uk/) (accessed on 22 November 2021).

\subsection{Statistical Analysis}

Data are shown as means \pm standard deviation. For single comparisons, significant differences between the means were determined by a one-way ANOVA Ordinary test. Statistical significance was set at $p<0.05$. All data were processed using GraphPad Prism 7 software (GraphPad Software, La Jolla, CA, USA).

Supplementary Materials: The following supporting information can be downloaded at: https: / / www.mdpi.com/article/10.3390/metabo12020121/s1, 1. Supplementary methods of Plasma sample preparation, UPLC-MS Analysis for metabolites, 2. Related information of the metabolites of HEP in MS data, 3. Data of intergrative analysis.

Author Contributions: G.B. and Y.H. (Yuanyau Hou) conceived and designed the experiments; H.W., L.T. and Y.H. (Yiman Han) conducted the experiments; H.W. and X.M. analyzed the data; H.W., G.B. and Y.H. (Yuanyau Hou) wrote and revised the manuscript. All authors have agreed to the published version of the manuscript.

Funding: This study was partly supported by the National Natural Science Foundation of China (no. 81973449). 
Institutional Review Board Statement: The animal protocols were approved by the Guide for the Ethical Use of the Guangxi University of Chinese Medicine (no. DW20200711-098, date of approval: 1 August 2020).

\section{Informed Consent Statement: Not applicable.}

Data Availability Statement: All data presented in this study are available from the authors upon request.

Acknowledgments: This work was supported by the Liaoning Multi-dimensional Analysis of Traditional Chinese Medicine Technical Innovation Center of the Mass Spectrometry Core Facility.

Conflicts of Interest: The authors declare no conflict of interest.

\section{References}

1. Desborough, M.J.R.; Keeling, D.M. The aspirin story-from willow to wonder drug. Br. J. Haematol. 2017, 177, 674-683. [CrossRef] [PubMed]

2. $\quad \mathrm{Su}, \mathrm{X} . ;$ Zhu, Z.-H.; Zhang, L.; Wang, Q.; Xu, M.-M.; Lu, C.; Zhu, Y.; Zeng, J.; Duan, J.-A.; Zhao, M. Anti-inflammatory property and functional substances of Lonicerae Japonicae Caulis. J. Ethnopharmacol. 2020, 267, 113502. [CrossRef] [PubMed]

3. Shang, X.; Pan, H.; Li, M.; Miao, X.; Ding, H. Lonicera japonica Thunb.: Ethnopharmacology, phytochemistry and pharma-cology of an important traditional Chinese medicine. J. Ethnopharmacol. 2011, 138, 1-21. [CrossRef] [PubMed]

4. Newman, D.J.; Cragg, G.M. Natural products as sources of new drugs from 1981 to 2014. J. Nat. Prod. 2016, 79, 629-661. [CrossRef] [PubMed]

5. Thomford, N.E.; Senthebane, D.A.; Rowe, A.; Munro, D.; Seele, P.; Maroyi, A.; Dzobo, K. Natural Products for Drug Discovery in the 21st Century: Innovations for Novel Drug Discovery. Int. J. Mol. Sci. 2018, 19, 1578. [CrossRef]

6. Chen, Y.; Johannes, K. Cheminformatics in Natural Product-based Drug Discovery. Mol. Inform. 2020, 39, e2000171. [CrossRef]

7. Pomyen, Y.; Wanichthanarak, K.; Poungsombat, P.; Fahrmann, J.; Grapov, D.; Khoomrung, S. Deep metabolome: Applications of deep learning in metabolomics. Comput. Struct. Biotechnol. J. 2020, 18, 2818-2825. [CrossRef]

8. Kirchweger, B.; Rollinger, J.M. A Strength-Weaknesses-Opportunities-Threats (SWOT) Analysis of Cheminformatics in Nat-ural Product Research. Prog. Chem. Org. Nat. Prod. 2019, 110, 239-271.

9. Kim, E.K.; Choi, E.-J. Pathological roles of MAPK signaling pathways in human diseases. Biochim. Biophys. Acta (BBA)-Mol. Basis Dis. 2010, 1802, 396-405. [CrossRef]

10. Zhang, M.; Ma, X.; Xu, H.; Wu, W.; He, X.; Wang, X.; Jiang, M.; Hou, Y.; Bai, G. A natural AKT inhibitor swertiamarin targets AKT-PH domain, inhibits downstream signaling, and alleviates inflammation. FEBS. J. 2020, 287, 1816-1829. [CrossRef]

11. Boltana, S.; Sanhueza, N.; Donoso, A.; Aguilar, A.; Crespo, D.; Vergara, D.; Arriagada, G.; Morales-Lange, B.; Mercado, L.; Rey, S.; et al. The expression of TRPV channels, prostaglandin E2 and pro-inflammatory cytokines during behavioural fever in fish. Brain, Behav. Immun. 2018, 71, 169-181. [CrossRef] [PubMed]

12. Guha, M.; Mackman, N. LPS induction of gene expression in human monocytes. Cell. Signal. 2001, 13, 85-94. [CrossRef]

13. Blomqvist, A.; Engblom, D. Neural Mechanisms of Inflammation-Induced Fever. Neuroscientist 2018, 24, 381-399. [CrossRef] [PubMed]

14. McGuire, V.; Gray, A.; Monk, C.E.; Santos, S.; Lee, K.; Aubareda, A.; Crowe, J.; Ronkina, N.; Schwermann, J.; Batty, I.H.; et al. Cross Talk between the Akt and p38 Pathways in Macrophages Downstream of Toll-Like Receptor Signaling. Mol. Cell. Biol. 2013, 33, 4152-4165. [CrossRef]

15. Harikrishnan, H.; Jantan, I.; Haque, A.; Kumolosasi, E. Anti-inflammatory effects of Phyllanthus amarus Schum. \& Thonn. through inhibition of NF-KB, MAPK, and PI3K-Akt signaling pathways in LPS-induced human macrophages. BMC Complement. Altern. Med. 2018, 18, 224. [CrossRef]

16. Peti, W.; Page, R. Molecular basis of MAP kinase regulation. Protein Sci. 2013, 22, 1698-1710. [CrossRef]

17. Patil, C.; Zhu, X.; Rossa, C.J.; Kim, Y.J.; Kirkwood, K.L. p38 MAPK regulates IL-1 beta induced IL-6 expression through mRNA stability in osteoblasts. Immunol. Investig. 2004, 33, 213-233. [CrossRef]

18. Liu, X.; Ye, F.; Xiong, H.; Hu, D.N.; Limb, G.A.; Xie, T.; Peng, L.; Zhang, P.; Wei, Y.; Zhang, W.; et al. IL-1beta induces IL-6 production in retinal Muller cells predominantly through the activation of p38 MAPK/NF-kappaB signaling pathway. Exp. Cell. Res. 2015, 331, 223-231. [CrossRef]

19. Sinfield, J.K.; Das, A.; O’Regan, D.J.; Ball, S.G.; Porter, K.E.; Turner, N.A. p38 MAPK alpha mediates cytokine-induced IL-6 and MMP-3 expression in human cardiac fibroblasts. Biochem. Biophys. Res. Commun. 2013, 430, 419-424. [CrossRef]

20. Lucas, A.J.; Sproston, J.L.; Barton, P.; Riley, R.J. Estimating human ADME properties, pharmacokinetic parameters and likely clinical dose in drug discovery. Expert Opin. Drug Discov. 2019, 14, 1313-1327. [CrossRef]

21. Wang, Z.; Kim, U.; Liu, J.; Cheng, C.; Wu, W.; Guo, S.; Feng, Y.; Quinn, R.; Hou, Y.; Bai, G. Comprehensive TCM molecular networking based on MS/MS in silico spectra with integration of virtual screening and affinity MS screening for discovering functional ligands from natural herbs. Anal. Bioanal. Chem. 2019, 411, 5785-5797. [CrossRef] [PubMed]

22. Wang, Z.; Wu, W.; Guan, X.; Guo, S.; Li, C.; Niu, R.; Gao, J.; Jiang, M.; Bai, L.; Leung, E.L.; et al. 20(S)-Protopanaxatriol promotes the binding of P53 and DNA to regulate the antitumor network via multiomic analysis. Acta Pharm. Sin. B 2020, 10, 1020-1035. [CrossRef] [PubMed] 
23. Gao, W.; Wang, C.; Yu, L.; Sheng, T.; Wu, Z.; Wang, X.; Zhang, D.; Lin, Y.; Gong, Y. Chlorogenic Acid Attenuates Dextran Sodium Sulfate-Induced Ulcerative Colitis in Mice through MAPK/ERK/JNK Pathway. BioMed Res. Int. 2019, 2019, 6769789. [CrossRef] [PubMed]

24. Cheng, D.; Zhang, X.; Tang, J.; Kong, Y.; Wang, X.; Wang, S. Chlorogenic acid protects against aluminum toxicity via MAPK/Akt signaling pathway in murine RAW264.7 macrophages. J. Inorg. Biochem. 2019, 190, 113-120. [CrossRef]

25. Gao, Y.; Wang, F.-X.; Liu, Q.; Qi, Y.-D.; Wang, Q.-L.; Liu, H.-B. Comparison of anti-inflammatory effects of Lonicerae Japonicae Flos and Lonicerae Flos based on network pharmacology. Chin. Herb. Med. 2021, 13, 332-341. [CrossRef]

26. Wang, H.C.; Li, T.J.; Bao, Y.R.; Wang, S.; Meng, X.S. Qualitative, quantitative, and pharmacokinetic study on the absorbed components of Ardisia japonica (Thunb.) Blume in rat plasma based on molecular networking combined with quadrupole time-of-flight LC/MS and triple quadrupole LC/MS. Biomed. Chromatogr. 2021, 35, e5099. [CrossRef]

27. Liu, X.; Ouyang, S.; Yu, B.; Liu, Y.; Huang, K.; Gong, J.; Zheng, S.; Li, Z.; Li, H.; Jiang, H. PharmMapper server: A web server for potential drug target identification using pharmacophore mapping approach. Nucleic Acids Res. 2010, 38, W609-W614. [CrossRef] 